\title{
GLuskap: Visualization and Manipulation of Graph Drawings in 3-Dimensions ${ }^{\star}$
}

\author{
Breanne Dyck, Jill Joevenazzo, Elspeth Nickle, Jon Wilsdon, and \\ Stephen Wismath \\ Department of Mathematics and Computer Science, University of Lethbridge, \\ Alberta, Canada T1K 3M4.
}

\begin{abstract}
GLuskap is a software tool for displaying graphs in 3 dimensions, interactively editing the resulting drawing and finally creating a high quality ray traced image. The software is written in $\mathrm{C}++$ and uses OpenGL and SDL. The interactive editing and navigation features may also be used in stereoscopic mode providing an even more realistic environment. The drawing can be exported either to a standard graph format or to source code directly suppliable to the POVRay ray tracing package which then renders the drawing into any standard image format. This software should be of value to all researchers in the 3D graph drawing community and is freely available at: http://www.cs.uleth.ca/ ${ }^{\sim} \mathrm{vpak} /$ demo.
\end{abstract}

\section{Overview}

Drawing graphs in 3 dimensions (3D) is a recent active research area. Whereas 2dimensional graph drawing has been supported by a variety of powerful software tools for some time, there are only a few 3D graph drawing packages and most are tailored for specific applications. GLuskap 1 is a software package for interactively accomplishing the creative and exploratory graph drawing and modeling commonly required by practioners in this area, and providing a general utility for the manipulation and visualization of 3-dimensional drawings of graphs.

GLuskap serves as an easy-to-use graphical user interface that produces 3dimensional graph drawings in OpenGL. See figure 1 The user is presented with numerous options for exploring 3D graph drawings, as well as having the ability to freely modify the representations. Of particular importance is the real-time interactive vertex and edge manipulation, which allows the user to modify the graph drawing, and immediately see the results in 3D. Import and export options accommodate various popular file formats, including GML and GraphML. A distinguishing feature of GLuskap is its ability to export a 3D graph drawing to the POV-Ray software package for subsequent rendering into a standard image format.

\footnotetext{
* Research supported by the Natural Sciences and Engineering Research Council of Canada.

${ }^{1}$ Gluskap is the Algonquin name for the Creator force 


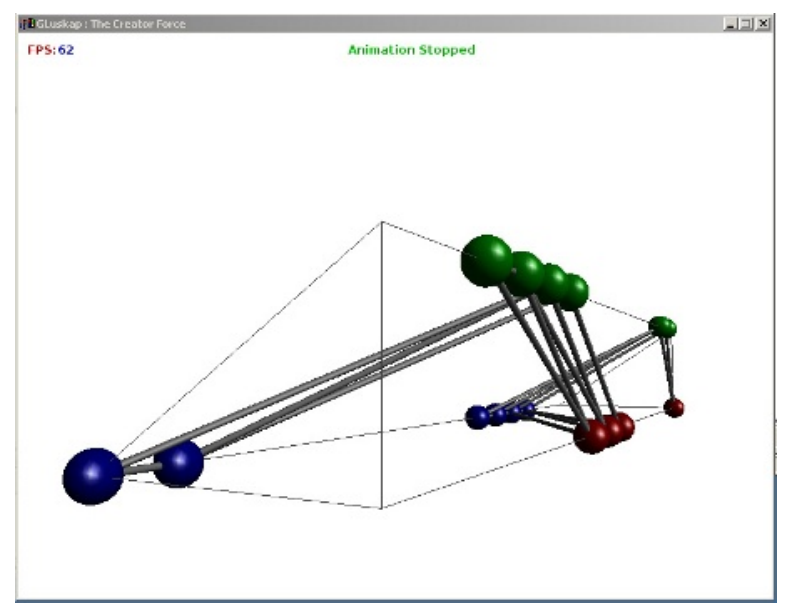

Fig. 1. Screenshot of GLuskap

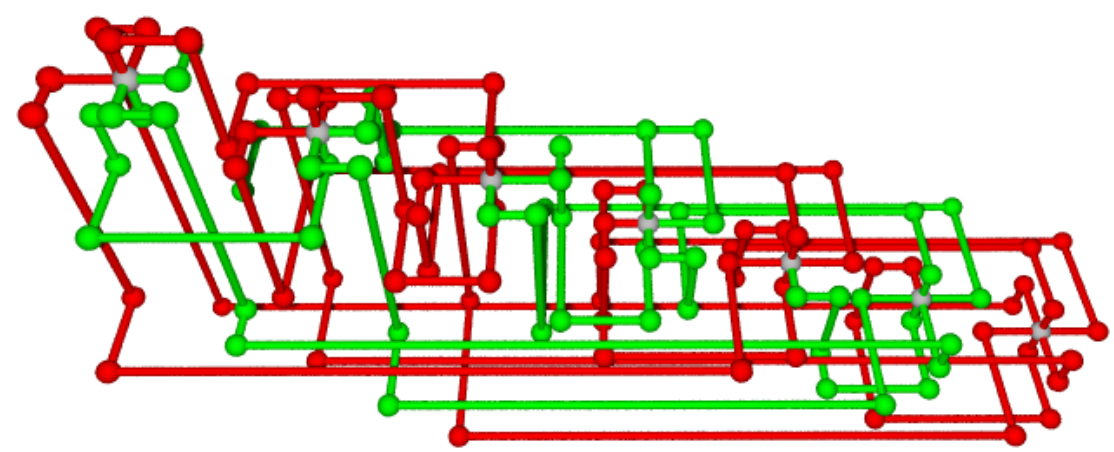

Fig. 2. A Ray-traced image

Figure 2 shows a rendered drawing produced through this process. Many users may wish to bypass the graphical editing/navigating process and concentrate solely on this post-processing step of creating POV-Ray source code. Nevertheless, the navigation aspects of the software are unique, as they provide interactive, 3-dimensional exploration of a graph drawing. Coupled with support for stereoscopic viewing, these features make the visualization aspects of GLuskap especially powerful. GLuskap is available for download as Windows executables and as $\mathrm{C}++$ source code from http://www.cs.uleth.ca/ ${ }^{\sim}$ vpak/demo. See the web site for further screenshots and more detailed information. 